\title{
Application of Uniform Design Genetic Algorithm in Separation Condition Optimization of Ginkgo Biloba Extract
}

\author{
Xueguo Chen* \\ Department of Forensic Chemistry, National Police University of China, Liaoning Shenyang 110854 \\ dicpchenxg@hotmail.com
}

\begin{abstract}
Objective: aiming to establish a new strategy based on uniform design and genetic algorithm and applying in the optimization of the separation condition of Ginkgo biloba extract using reversed-phase liquid chromatography according to traditional optimization methods. Methods: based on uniform design and genetic algorithm, a new strategy was developed and a software package was developed. The optimization strategy was applied in the optimization of separation condition of Ginkgo biloba extract in reversed-phase chromatography, the volume fraction of acetonitrile and rate of linear gradient-elution were selected as the optimization variables. Results: The optimization of separation condition of Ginkgo biloba extract in reversed-phase chromatography was performed and satisfactory optimal result was obtained as the initial concentration of acetonitrile was $14.4 \%$ and the rate of linear gradient-elution was $0.22 \%$, respectively. Conclusion: The results shown that the method using uniform design and genetic algorithm is efficient and it has potential application not only in the optimization of two parameters, but also of much more than two parameters.
\end{abstract}

Keywords-genetic algorithm; liquid chromatography; condition optimization; Ginkgo biloba extract; linear gradient-elution

\section{INTRODUCTION}

With the development of theory, technique and instruments, high performance liquid chromatography has become the most efficient separation tool in the analysis of complex samples such as environmental samples, biological samples, medical samples and medical quality control ${ }^{[1,2]}$. The complexities of real samples have showed the necessity of optimization of chromatographic separation conditions ${ }^{[3]}$.

Genetic algorithm is an optimization algorithm that mimics the mechanisms of natural selection described by genetics and the Darwinian theory of evolution ${ }^{[4,5]}$. As global searching algorithm, genetic algorithm makes use of artificial intelligence to obtain the solution of rather complex problems, so it has been widely applied in resolving combinatorial optimization problems ${ }^{[6]}$, and attempter problems ${ }^{[7]}$ due to their parallelism and effective utilization of global information.
In this article, according to traditional optimization methods, a new strategy based on uniform design and genetic algorithm was established. The optimization strategy was described in details and a software package was developed. The optimization strategy was applied in the optimization of separation condition of Ginkgo biloba extract in reversed-phase chromatography and satisfactory optimal result was obtained.

\section{OPTIMIZATION STRATEGY}

The outline of the optimizing process based on genetic algorithm is shown in Fig .1. The procedures including selection of column system, screening for mobile phase and components, searching for the optimization point of mobile phase, has been discussed in our past work ${ }^{[8]}$.

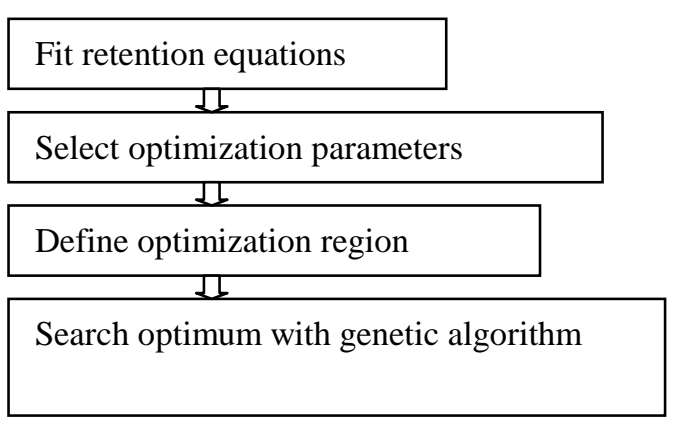

Figure 1. Outline of the principle of optimization strategy

In this article, two parameters, the initial concentration of acetonitrile and the rate of linear gradient-elution, were chosen to be optimized. Thus two-dimensional points were selected to denote the individuals and the coordinates of these points denote the initial concentration of acetonitrile and the rate of linear gradient-elution in our system, and they were encoded in real number. The procedures in details is shown in Fig .2 and can be expressed as following:

\section{A. Initialization}

Genetic algorithm does not operate on an individual for searching the parameter space but on a group of points (called population) at a time. Generally, an initial 
group includes 20 to 300 points. In this work, an initial group containing 30 two-dimensional points was generated with the uniform design method ${ }^{[9]}$ in the region of optimization parameters.

\section{B. Line-crossover}

Line-crossover was the major operation of genetic algorithm; the detail is illustrated in Fig .3. It is supposed that $\mathrm{A}$ and $\mathrm{B}$ are the cross points. Drawing a line between $\mathrm{A}$ and $\mathrm{B}$, setting the midpoint of $\mathrm{AB}$ as $\mathrm{C}$, then extending $\mathrm{BA}$ to $\mathrm{D}$, where $\mathrm{A}$ is the midpoint of $\mathrm{CD}$, then extending $A B$ to $E$, just fitting $B$ is the midpoint of CE. After these operations, three points, which are offspring of $\mathrm{A}$ and $\mathrm{B}$, are obtained. In this work, the points in the initial group were divided into 15 units, after line-crossover accomplished, the number of the group was change to 45 points.

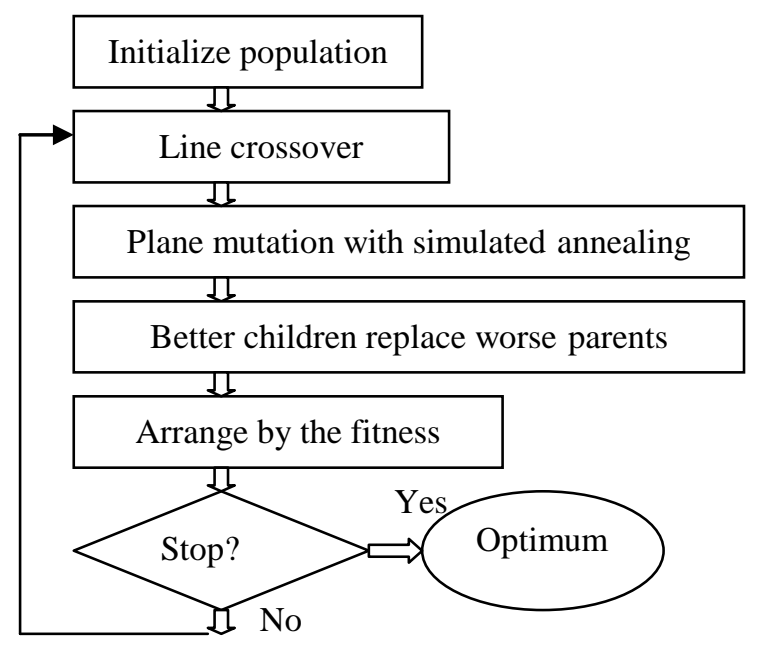

Figure 2. Outline of the process of genetic algorithm

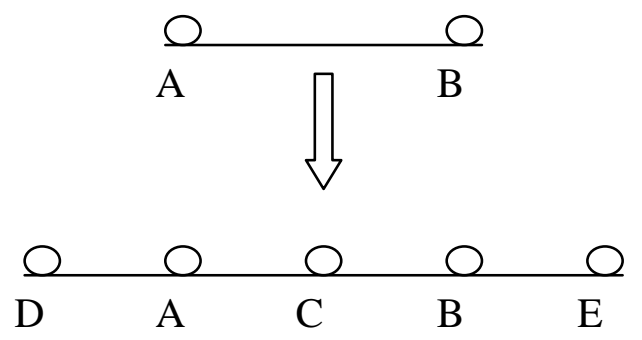

Figure 3. Points A, B and their crossover children points C, D and E

\section{Plane-mutation}

The major operation of plane-mutation is subtracting and adding of variables. It is supposed that $\mathrm{H}$ $\left(\mathrm{C}_{\mathrm{M}}, \mathrm{C}_{\mathrm{T}}\right)$ is the point before mutation, and $\mathrm{J}\left(\mathrm{C}_{\mathrm{M}}, \mathrm{C}_{\mathrm{T}}\right)$ is the point that after mutation, thus $\mathrm{J}=\mathrm{H}+\mathrm{A}$, where $\mathrm{A}$ is mutation range $(A=\{a 1, a 2\})$. In addition, mutation range should be reduced after once mutation, $A^{\prime}$ is defined as the new mutation range after mutation, thus $\mathrm{A}^{\prime}=\alpha \times \mathrm{A}$, where $\alpha$ is the reduced coefficient. The value of the reduced coefficient is 0.99 in general. In this work, because every individual is a two-dimensional point, the operation of plane-mutation was illustrated in Fig .4 in detail. It is supposed that $\mathrm{O}$ is the plane-mutation point, after plane-mutation, eight points, which are offspring of $\mathrm{O}$, are obtained. The numbers of the group increased to 360 after this operation.

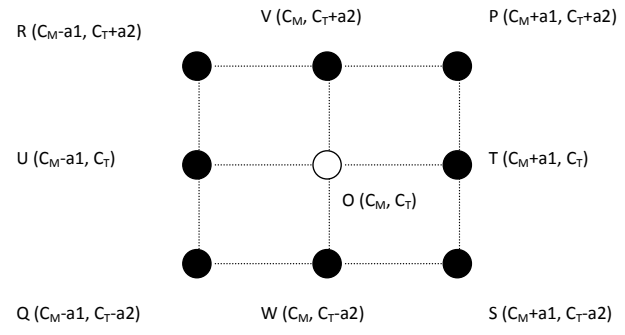

Figure 4. Uniform design settings for the 7 starting experiments

\section{Arranging with the fitting values}

The retention time and peak widths at half height of all solutes are firstly predicted according to Equation (1) and Equation (2) with CM and CT denoted by the points (offspring) to be arranged.

$$
\begin{aligned}
& t_{R}{ }^{\prime}=-\frac{\ln \left(1-c \times G \times t_{0} \times e^{a+c \times C_{B 0}}\right)}{c \times G} \\
& \mathrm{~W}_{1 / 2}=\mathrm{a}_{\mathrm{w}}+\mathrm{b}_{\mathrm{w}} \times t_{R}
\end{aligned}
$$

Then the resolution (Rs) ${ }^{[10]}$ of neighbor pair peaks can be counted. If Rs $>1.0$, it means two solutes can be separated according to the Rs value. Otherwise, two solutes can not be resolved if $\mathrm{Rs}<1.0$. A hierarchical chromatographic response function (HCRF) ${ }^{[1]}$ is modified and used to evaluate the quality of a chromatogram as follows:

$$
H C R F=1000000 \times \mathrm{n}+100000 \times \mathrm{R}_{\mathrm{s}_{\text {min }}}+\left(T_{D}-T_{L}\right)
$$

where $\mathrm{n}$ is the number of resolved peaks in the chromatogram. Rsmin is the resolution for the least resolved pair of peaks (when Rsmin $>1.60$, which means that all of components can be baseline-separated, then Rsmin is specified as 1.60). $T_{D}$ and $T_{L}$ are the maximum acceptable analysis time and the retention time of the last peak, respectively. If $T_{L}$ is larger than $T_{D}$, it means that the analysis time is too long to be accepted, and then HCRF is specified as 1000000.00 . Finally, all the points are arranged according to their HCRF value.

\section{E. Replacement of worse parents by better children}

Certain numbers of better children are chosen to replace certain numbers of worse parents, which can keep parents in certain number and make parents evolved. In this work, 5 better children were chosen to replace 5 worse parents.

According to the stop criterion of genetic algorithm calculation, it will be stopped if the best individual is not replaced for 20 times.

\section{EXPERIMENTAL}

\section{A. Apparatus}

Waters HPLC 515 pump and 2487 dual $\lambda$ absorbance detector (Waters, USA); WDL-95 
workstation (National Chromatographic R. \& A. Center, China).

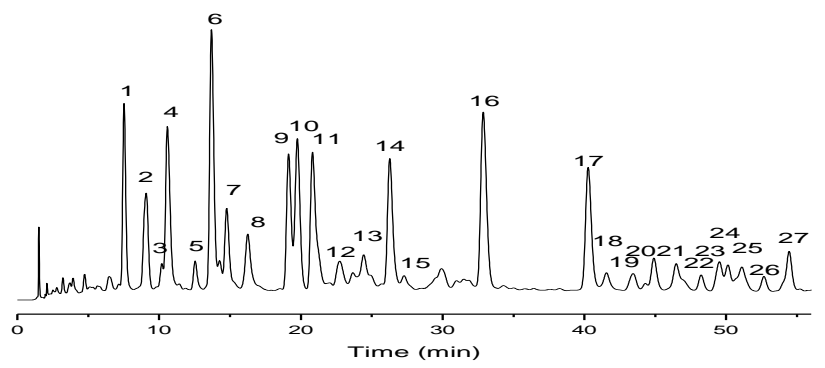

Figure 5. Chromatogram of Ginkgo biloba extract under the optimal conditions

TABLE I. PREDICTED (TP) AND EXPERIMENTAL (TE) RETENTION TIME OF GINKGO BILOBA EXTRACT AT THE OPTIMUM AND RELATIVE DIFFERENCE (RD) BETWEEN THEM

\begin{tabular}{|c|c|c|c|}
\hline \multirow{2}{*}{$\begin{array}{l}\text { Component } \\
\text { code }\end{array}$} & \multicolumn{3}{|c|}{ Linear gradient elution } \\
\hline & $\mathrm{t}_{\mathrm{e}} / \mathrm{min}$ & $\mathrm{t}_{\mathrm{p}} / \min$ & $\mathrm{RD}(\%)$ \\
\hline 1 & 7.53 & 7.33 & 2.66 \\
\hline 2 & 9.09 & 8.72 & 4.07 \\
\hline 3 & 10.19 & 9.81 & 3.73 \\
\hline 4 & 10.58 & 10.20 & 3.59 \\
\hline 5 & 12.54 & 12.04 & 3.99 \\
\hline 6 & 13.69 & 13.13 & 4.09 \\
\hline 7 & 14.77 & 14.18 & 3.99 \\
\hline 8 & 16.25 & 15.61 & 3.94 \\
\hline 9 & 19.13 & 17.90 & 6.43 \\
\hline 10 & 19.75 & 18.99 & 3.85 \\
\hline 11 & 20.82 & 20.32 & 2.40 \\
\hline 12 & 22.74 & 21.88 & 3.78 \\
\hline 13 & 24.43 & 23.83 & 2.46 \\
\hline 14 & 26.27 & 26.13 & 0.53 \\
\hline 15 & 27.28 & 27.70 & 1.54 \\
\hline 16 & 32.87 & 33.46 & 1.79 \\
\hline 17 & 40.26 & 39.82 & 1.09 \\
\hline 18 & 42.56 & 42.26 & 0.70 \\
\hline 19 & 43.43 & 43.59 & 0.37 \\
\hline 20 & 44.90 & 44.29 & 1.36 \\
\hline 21 & 46.48 & 46.03 & 0.97 \\
\hline 22 & 48.24 & 48.18 & 0.12 \\
\hline 23 & 49.52 & 50.29 & 1.55 \\
\hline 24 & 50.12 & 52.67 & 5.09 \\
\hline 25 & 51.10 & 53.76 & 5.21 \\
\hline 26 & 52.66 & 54.42 & 3.34 \\
\hline 27 & 54.44 & 55.88 & 2.65 \\
\hline $\mathrm{RD}_{\text {max }}$ & & 6.43 & \\
\hline $\mathrm{RD}_{\text {ave }}$ & & 2.79 & \\
\hline
\end{tabular}

The separations were carried out on an ODS column $(150 \times 4.6 \mathrm{~mm}$ i.d., $5 \mu \mathrm{m})$; mobile phase were acetonitrile and $0.10 \%$ acetic acid with linear gradient-elution, the initial concentration of acetonitrile was $14.4 \%$ and the rate of linear gradient-elution was $0.22 \%$; the flow rate of mobile phase was $1.0 \mathrm{~mL} / \mathrm{min}$; detection wavelength was set as $350 \mathrm{~nm}$ and the injection volume was $10 \mu \mathrm{L}$.

B. Reagent

Acetonitrile and acetic acid were both of RP grade and purchased from Shenyang Regent Factory (Shenyang, China); Ginkgo biloba extract was obtained from Dalian Refine Co. Ltd. (Dalian, China).

IV. RESULTS AND DISCUSSION

According to reports in literatures ${ }^{[11]}$ and our past work ${ }^{[12]}$, in this experiment reversed-phase high-performance liquid chromatography with ODS column was chosen as the separation mode for Ginkgo biloba extract. The mobile phase was composed of acetonitrile and $0.10 \%$ acetic acid. The volume fraction of acetonitrile and rate of linear gradient-elution were selected the optimization variables, the ranging were from 0 to $60 \%$ (v\%) and 0 to $1 \%$, respectively. The optimum was obtained with the optimization strategy applied uniform design and genetic algorithm according to the principle of the optimization strategy as shown in Fig .2, the initial concentration of acetonitrile was $14.4 \%$ and the rate of linear gradient-elution was $0.22 \%$, respectively. The experimental results and predicted data are listed in Table 1 and the separation chromatogram of Ginkgo biloba extract under the optimal experimental conditions is listed in Fig .3, which confirmed that the accuracy to search the optimal condition was very high and the prediction conditions was satisfying.

Furthermore, the optimization method mentioned here has two characteristics. One is uniform design, which makes it possible that the starting experiments are uniformly located and the retention of solutes is more accurately predicted in the range of variables to be optimized. Therefore, the disadvantages of the sequential approaches such as simplex for the local optimum can be overcome. Another is genetic algorithm, which makes it possible to increase the accuracy for prediction of the migration times of solutes. Moreover, the global optimum can be obtained.

\section{$\mathrm{V}$. CONCLUSION}

The optimization method using uniform design and genetic algorithm was applied in optimizing the chromatographic separation in reversed-phase chromatography mode. The process of optimizing the content of acetonitrile in the mobile phase and the rate of linear gradient-elution using uniform design and simulated annealing genetic algorithm was introduced in detail. Ginkgo biloba extract was selected to test the optimization method and the optimum was obtained by optimization procedures, the mean relative deviation between predicted and experimental values was only $2.79 \%$ under the optimum separation condition. The results shown that the method using uniform design and 
genetic algorithm is efficient and it has potential application not only in the optimization of two parameters, but also of much more than two parameters.

\section{REFERENCES}

[1] H. F. Zou, Y. K. Zhang, P. Z. Lu. Ion Pair High Performance Liquid Chromatography. Henan: Henan Science and Technology Press, 1994. 108.

[2] L. H. Buenno, J. Leme, C. P. Caricati, A. Tonso, J. C. Rocha, E. G. Fernández Núñez. Use of uniform designs in combination with neural networks for viral infection process development. Biotechnol Prog. 2015 doi: 10.1002/btpr.2051

[3] W. Y. Li, H. T. Rasmussen. Strategy for developing and optimizing liquid chromatography methods in pharmaceutical development using computer-assisted screening and Plackett-Burman experimental design. Journal of Chromatography A, 2003, 1016 (2): 165-180

[4] L. Yu, S. R. Wang, Y. Qu, G. Y. Lin. Broadband FUV imaging spectrometer: advanced design with a single toroidal uniform-line-space grating. Appl Opt. 2011, 50 (22): 4468-4477.

[5] J. F. Li, H. Liao, B. Normand, C. Cordier, G. Maurin, J. Foct, C. Coddet. Uniform design method for optimization of process parameters of plasma sprayed TiN coatings. Surface \& Coatings Technology, 2003, 176 (1): 1-13.

[6] M. Novokmet, M. Pučić, I. Redžić, A. Mužinić, O. Gornik. Robustness testing of the high throughput HPLC-based analysis of plasma N-glycans. Biochim Biophys Acta. 2012, 1820 (9): 1399-1404.
[7] K.M. Rehana, K.S. Beng. Optimization of the simultaneous batch determinations of $\mathrm{Bi}$ (III), $\mathrm{Hg}$ (II) and $\mathrm{Cu}$ (II) at an epoxy-graphite electrode bulk modified with 2-mercaptobenzothiazole. Analyst, 2001, 126 (12): 2172-2177.

[8] X. G. Chen, X. Li, L. Kong, J. Y. Ni, R. H. Zhao, H. F. Zou. Application of uniform design and genetic algorithm in optimization of reversed-phase chromatographic separation. Chemometrics and Intelligent Laboratory Systems, 2003, 67: 157-166.

[9] R. J. Lee, Z. Liu, H. F. Zou, J. Y. Ni, Y. K. Zhang. Multivariate optimization in micellar electrokinetic capillary chromatography. Journal of Microcolumn seperation, 2000, 12: 356-365.

[10] H. F. Zou, Y. K. Zhang, P. C. Lu. Ion Pair High Performance Liquid Chromatography. Henan, Science and Technology Press, Henan, 1994.

[11] P. C. Lu, H. X. Huang. An intelligent search method for HPLC optimization. Journal of Chromatography Science, 1989, 27: 690-697.

[12] M. Zecevic, L. Zivanovic, S. Agatonovic-Kustrin, D. Minic. The use of a response surface methodology on HPLC analysis of methyldopa, amiloride and hydrochlorothiazide in tablets. Journal of Pharmaceutical and Biomedical Analysis, 2001, 24 (5-6): 1019-1025.

[13] X. G. Chen, R. H. Zhao, J. Y. Ni, H. F. Zou. Application of simulated annealing genetic algorithm in optimization of linear gradient-elution conditions in high performance liquid chromatography. Chinese Journal of Chromatography, 2004, 22 (1): 87. 